\title{
Studies of particle production in inelastic $p p$ events with the ATLAS detector
}

\author{
Ben Wynne for the ATLAS Collaboration \\ University of Edinburgh, Mayfield Rd, Edinburgh, EH9 3JZ \\ DOI: http://dx.doi.org/10.5689/UA-PROC-2010-09/24
}

\begin{abstract}
The charged-particle density, transverse momentum and pseudorapidity distributions are measured in proton-proton interactions at $\sqrt{s}=900 \mathrm{GeV}, 2.36 \mathrm{TeV}$ and $7 \mathrm{TeV}$ with the ATLAS detector. These charged-particle distributions are used to create a new PYTHIA6 tune. $K_{\mathrm{S}}^{0}$ mesons and $\Lambda_{0}$ baryons are identified and their properties measured.
\end{abstract}

\section{Introduction}

The ATLAS detector consists of several subdetectors built as coaxial cylinders, centred on and enclosing one of the four 'interaction points' of the LHC, where the proton beams collide. In December 2009, ATLAS recorded $p p$ collisions at $\sqrt{s}=0.9$ and $2.36 \mathrm{TeV}$, with $7 \mathrm{TeV}$ collisions beginning in March 2010. Beam pickups are attached to the beam-pipe at $\pm 175 \mathrm{~m}$ and are used to define crossing proton bunches. Trigger scintillators cover the region $2.09<|\eta|<3.84$, separated into two units in pseudorapidity and two in azimuth - activity in any of these during a bunch crossing is regarded as a potential collision.

Tracking is provided by three layers of silicon pixels closest to the beam, four double layers of single-sided silicon strips and 73 layers of straw tubes, all surrounded by a solenoid providing a $2 \mathrm{~T}$ axial magnetic field for momentum resolution. Using the tracker, distributions of charged particles from these early $p p$ collisions have been measured and compared with predictions from Monte Carlo event generators. See Section 2 for these comparisons, and Section 3 for the creation of a new event generator 'tune'. The tracker has also been used to reconstruct long lived, weakly decaying particles. The performance of the tracker was studied by comparing the measured properties of these particles with their established values, as discussed in Section 4 .

\section{Event Selection}

Events were selected which contained a reconstructed vertex, which was formed by at least three tracks with $p_{\mathrm{T}}>150 \mathrm{MeV}$. If there was another primary vertex with four or more tracks, the event was rejected. Each track used in the analysis must have $p_{\mathrm{T}}>500 \mathrm{MeV}$, at least one silicon pixel hit and six silicon strip hits, and must have longitudinal and transverse impact parameters $<1.5 \mathrm{~mm}$ with respect to the primary vertex. If no tracks passed these criteria the event was rejected. No attempt was made to correct for the detector acceptance and the result is presented within $|\eta|<2.5$. Using these selections, charged-particle distributions have been measured at $\sqrt{s}=0.9$ and $7 \mathrm{TeV}[1,2]$. Slightly modified selections were required at 
$\sqrt{s}=2.36 \mathrm{TeV}$, because the silicon detectors were run at lower voltage for these collisions [3]. Example distributions from collisions at these three energies are shown in Figure 1. A similar analysis within the kinematic range of $p_{\mathrm{T}}>100 \mathrm{MeV}$ and $|\eta|<2.5$ was performed [4].

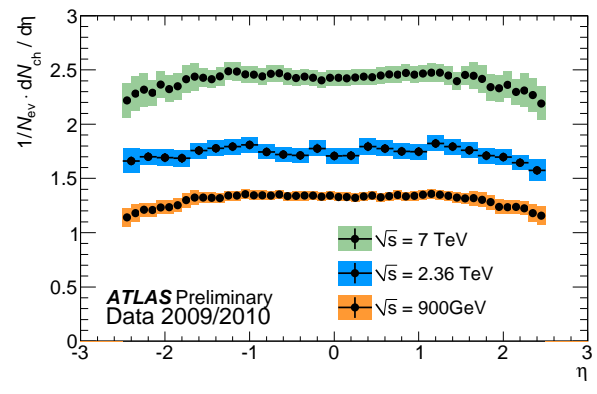

(a) Charged-particle density vs pseudorapidity

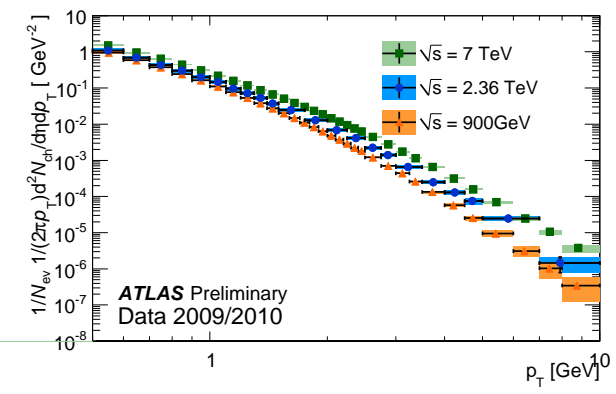

(b) Charged-particle density vs transverse momentum

Figure 1: Distributions of charged particles with $|\eta|<2.5$, for events with at least one charged particle $\left(n_{\mathrm{ch}} \geq 1\right)$ at $\sqrt{s}=0.9,2.36$ and $7 \mathrm{TeV}$. Shaded regions show combined statistical and systematic errors.

\section{Tuning Pythia}

A new 'tune' of Pythia6 (version 6.4.21) [5] was produced to better describe the data; this tune is referred to as ATLAS Minimum Bias Tune 1 (AMBT1). Existing CDF and D0 data were used for tuning, as well as the new ATLAS charged-particle distributions [6]. Events with fewer than six tracks passing the selection were ignored to reduce contributions from diffractive processes, since these are not well modelled in PythiA6. Starting from the ATLAS MC09c tune, the following Pythia6 parameters were adjusted, using Professor [7] to select the best value within the given range ('fixed' parameters were set by hand):

\begin{tabular}{|l|c|c|c|c|}
\hline Parameter & Related model & MC09c value & Tuning range & AMBT1 value \\
\hline PARP $(62)$ & ISR cut-off & 1.0 & fixed & 1.025 \\
PARP $(93)$ & Primordial kt & 5.0 & fixed & 10.0 \\
PARP $(77)$ & CR suppression & 0.0 & $0.25-1.15$ & 1.016 \\
PARP $(78)$ & CR strength & 0.224 & $0.2-0.6$ & 0.538 \\
PARP $(83)$ & MPI (matter fraction in core) & 0.8 & fixed & 0.356 \\
PARP $(84)$ & MPI (core of matter overlap) & 0.7 & $0.0-1.0$ & 0.651 \\
PARP $(82)$ & MPI ( $p_{T}^{\text {min }}$ ) & 2.31 & $2.1-2.5$ & 2.292 \\
PARP $(90)$ & MPI (energy extrapolation) & 0.2487 & $0.18-0.28$ & 2.50 \\
\hline
\end{tabular}

Table 1: PythiA6 parameters in AMBT1 that were changed compared to MC09c.

AMBT1 produces a larger number of charged particles than MC09c, and has a softer trans- 
verse momentum spectrum. The following plots compare AMBT1 with ATLAS 7 TeV chargedparticle distribution, showing better agreement than earlier models.

While AMBT1 describes the data well when only including tracks with $p_{\mathrm{T}}>500 \mathrm{MeV}$, it does little better than any of the other PYтніa6 tunes when including tracks with $p_{\mathrm{T}}>100 \mathrm{MeV}$, since the Pythia6 model itself cannot describe these events. Figure 2 shows the chargedparticle density is consistently underestimated at all ${ }^{1}$ available LHC centre-of-mass energies.

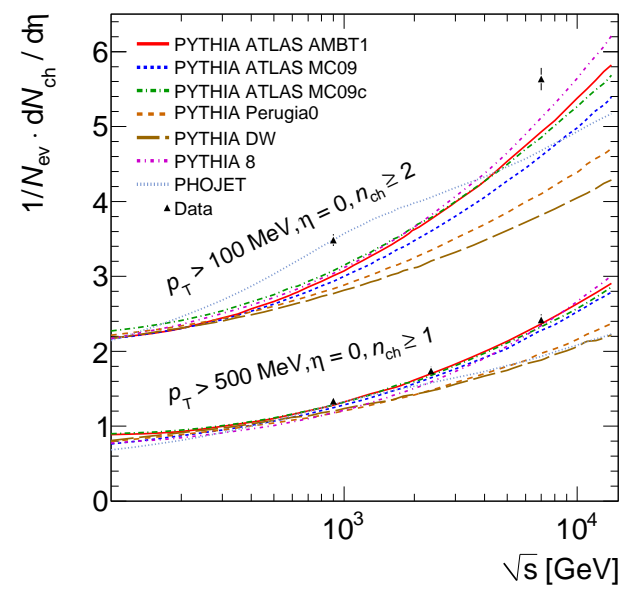

Figure 2: Comparing results from the charged-particle $\eta$ distributions with particle $p_{\mathrm{T}}$ minimum at $100 \mathrm{MeV}$, and at $500 \mathrm{MeV}$

\section{Identified Particles}

The detector performance and its agreement with an associated GEANT4 [8] simulation [9] was studied by reconstructing $K_{\mathrm{S}}^{0}$ (K-short) and $\Lambda_{0}$ particles [10].

$K_{\mathrm{S}}^{0}$ decays to $\pi^{+} \pi^{-}$with a $69 \%$ branching ratio [11] after a mean proper flight length of $27 \mathrm{~mm}$. $\Lambda_{0}$ decays to $\mathrm{p} \pi^{-}$with a $64 \%$ branching ratio after a mean proper flight length of $79 \mathrm{~mm}$. Therefore, events are selected if they contain a secondary vertex formed from two oppositely charged particles. Other constraints [10] ensure the quality of this vertex, and require it to be a minimum distance from the primary vertex. For $K_{\mathrm{S}}^{0}$ the minimum required distance is $4 \mathrm{~mm}$ perpendicular to the beam axis, or $30 \mathrm{~mm}$ required total distance for $\Lambda_{0}$.

Selecting events where the mass of the reconstructed vertex is within $20 \mathrm{MeV}$ of the PDG value for $K_{\mathrm{S}}^{0}$, or within $7 \mathrm{MeV}$ for $\Lambda_{0}$, the kinematic distributions in Figure 4 were produced. The same selections have also been applied to Monte Carlo events (generated with PyThIA6, using the ATLAS MC09 tune ${ }^{2}$ ). These events were passed through a simulation of the detector in GEANT4, and the ratio of signal to background was adjusted to best fit the data. Note the excellent agreement between the data from the detector and the simulated events shown in the following plots, suggesting that detector performance is well understood.

\footnotetext{
${ }^{1}$ The analysis for tracks with $p_{\mathrm{T}}>100 \mathrm{MeV}$ could not be performed for $\sqrt{s}=2.36 \mathrm{TeV}$

${ }^{2}$ not the same as MC09c mentioned in Section 3
} 


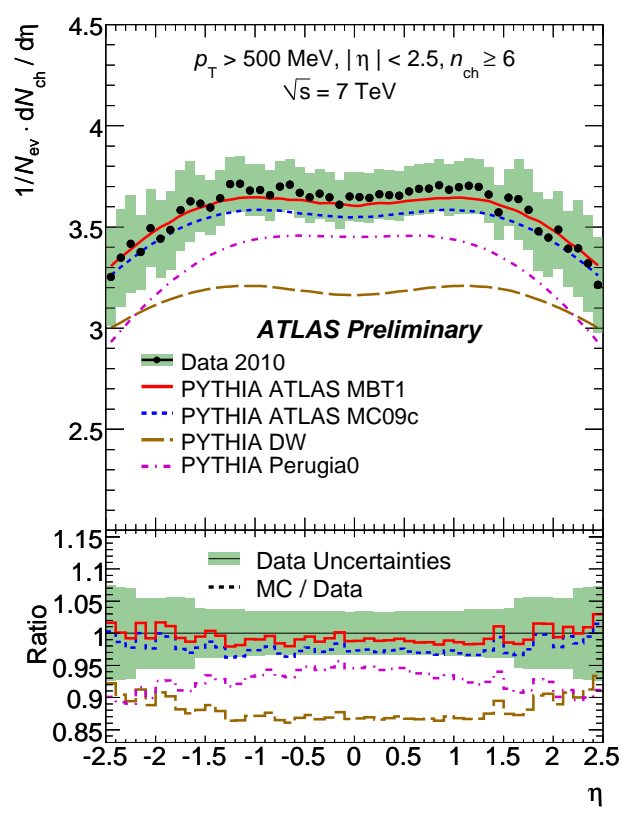

(a) Charged-particle density vs pseudorapidity

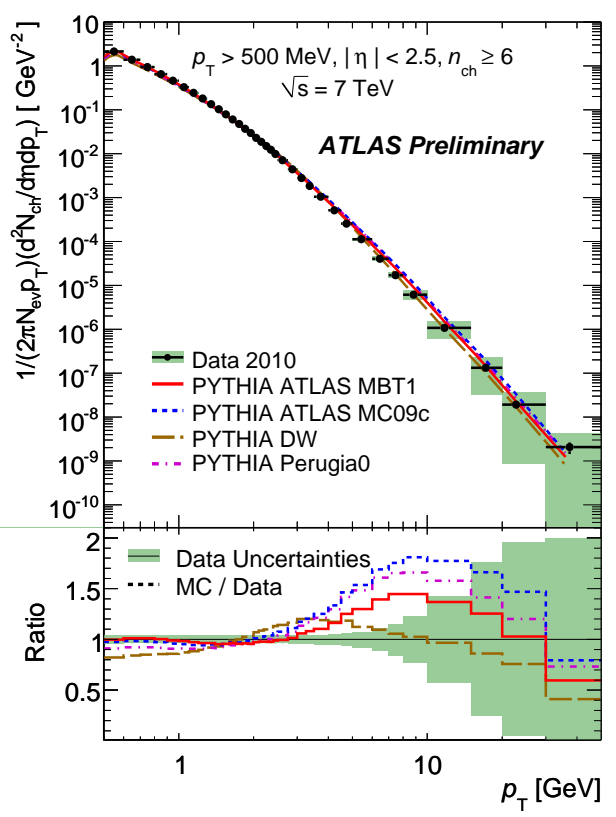

(c) Charged-particle density vs transverse momentum

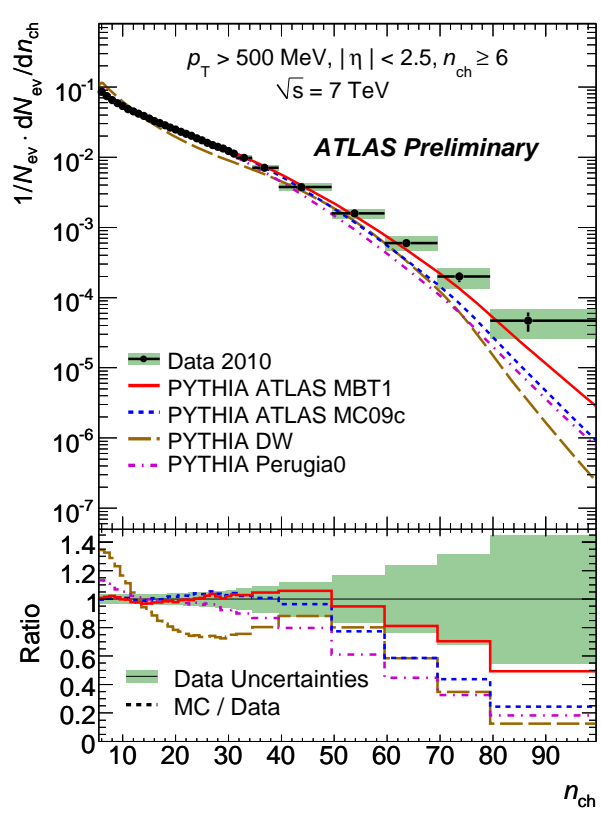

(b) Charged-particle multiplicity

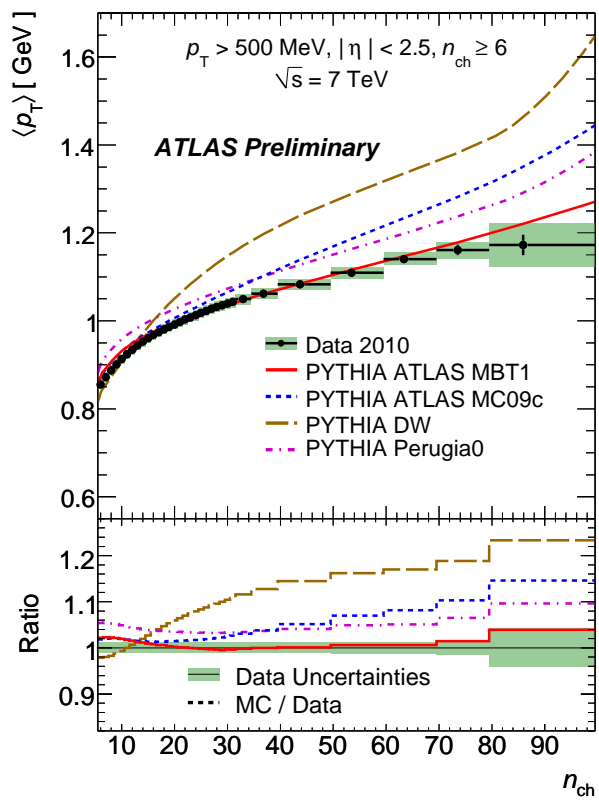

(d) Charged-particle mean transverse momentum

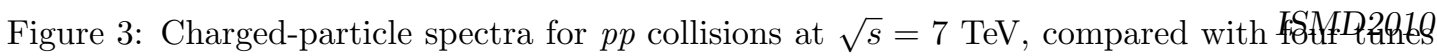
of Pyтнia6 


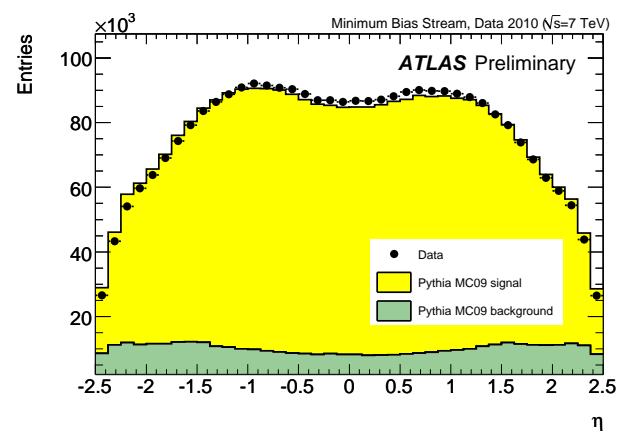

(a) $K_{\mathrm{S}}^{0}$ pseudorapidity distribution

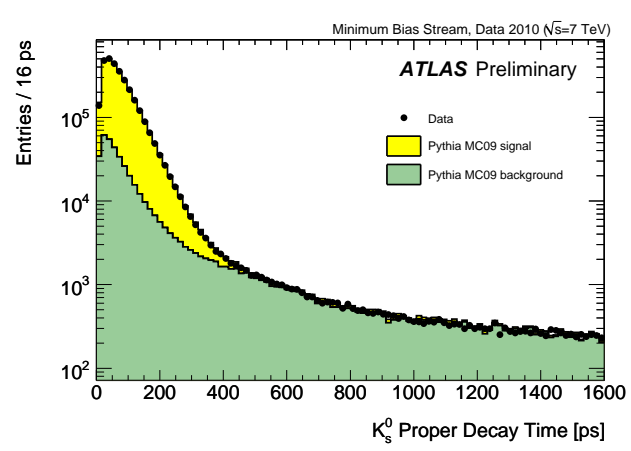

(c) $K_{\mathrm{S}}^{0}$ proper decay times

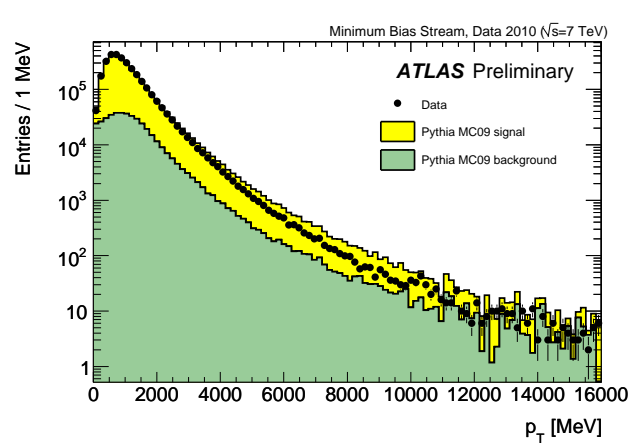

(e) $K_{\mathrm{S}}^{0}$ transverse momentum distribution

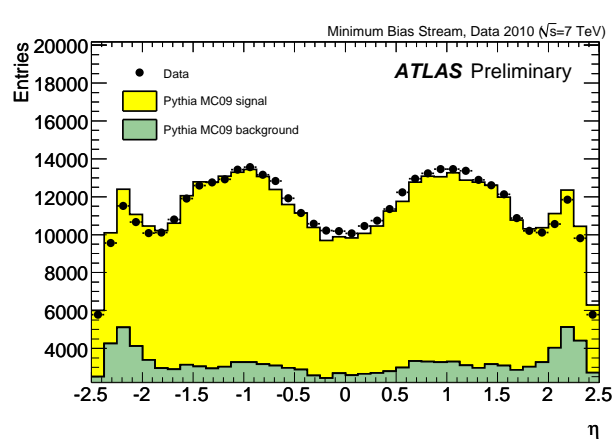

(b) $\Lambda_{0}$ pseudorapidity distribution

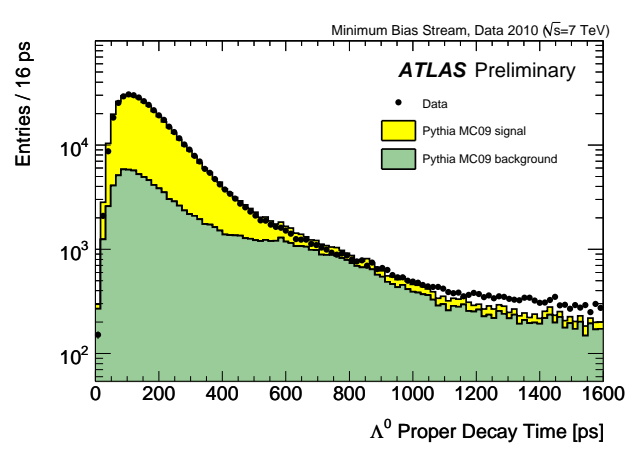

(d) $\Lambda_{0}$ proper decay times

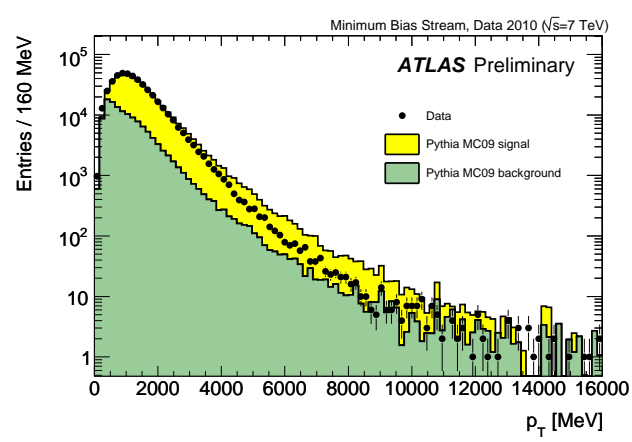

(f) $\Lambda_{0}$ transverse momentum distribution

Figure 4: Kinematic distributions for $K_{\mathrm{S}}^{0}$ and $\Lambda_{0}$ 


\section{Conclusion}

From early ATLAS data, distributions of charged particles in the detector have been constructed, and the properties of specific particles have been measured. The new PYTHIA6 tune AMBT1 gives an improved description of these charged-particle distributions compared with tunes created before ATLAS data was available. Using the selections described - except the mass constraint on the secondary vertex - mass spectra for the $K_{\mathrm{S}}^{0}$ and $\Lambda_{0}$ candidate particles have been constructed. Measured detector performance agrees with that predicted by simulation, and reconstructed $K_{\mathrm{S}}^{0}$ and $\Lambda_{0}$ particle masses are consistent with their established values.

\section{Acknowledgements}

Naturally these results depend on work by the entire ATLAS Collaboration, but my particular thanks to Will Bell, Jed Biesiada, Andy Buckley and Emily Nurse for their help preparing this document, and to the organisers of ISMD 2010.

\section{References}

[1] G. Aad et al. [ATLAS Collaboration], Phys. Lett. B 688 (2010) 21 [arXiv:1003.3124 [hep-ex]].

[2] [ATLAS Collaboration], "Charged particle multiplicities in $p p$ interactions at $\sqrt{s}=7 \mathrm{TeV}$ measured with the ATLAS detector at the LHC," ATLAS-CONF-2010-024.

[3] [ATLAS Collaboration], "Charged particle multiplicities in $p p$ interactions at $\sqrt{s}=2.36 \mathrm{TeV}$ measured with the ATLAS detector at the LHC," ATLAS-CONF-2010-047.

[4] [ATLAS Collaboration], "Charged particle multiplicities in $p p$ interactions for track $p_{\mathrm{T}}>100 \mathrm{MeV}$ at $\sqrt{s}=0.9$ and $7 \mathrm{TeV}$ measured with the ATLAS detector at the LHC," ATLAS-CONF-2010-046.

[5] T. Sjostrand, S. Mrenna, and P. Skands, JHEP 05 (2006) 026 [hep-ph/0603175].

[6] [ATLAS Collaboration], "Charged particle multiplicities in $p p$ interactions at $\sqrt{s}=0.9$ and $7 \mathrm{TeV}$ in a diffractive limited phase space measured with the ATLAS detector at the LHC and a new PYTHIA6 tune," ATLAS-CONF-2010-031.

[7] Buckley, A.; Hoeth, H.; Lacker, H.; Schulz, H.; von Seggern, J., Eur. Phys. J C65:331-357 (2010) [arXiv:0907.2973v1[hep-ph]].

[8] S. Agostinelli et al. [GEANT4 Collaboration], Nucl. Instr. Meth. A506:250-303 (2003).

[9] Z. Marshall [ATLAS Collaboration], "The ATLAS simulation software," ATL-SOFT-PROC-2008-001.

[10] [ATLAS Collaboration], "Kinematic Distributions of $K_{\mathrm{S}}^{0}$ and $\Lambda_{0}$ decays in collision data at $\sqrt{s}=7 \mathrm{TeV}$," ATLAS-CONF-2010-033.

[11] K. Nakamura et al. [Particle Data Group], JPG 37, 075021 (2010) [URL: http://pdg.lbl.gov] 